“C 2019 IEEE. Personal use of this material is permitted. Permission from IEEE must be obtained for all other uses, in any current or future media, including reprinting/republishing this material for advertising or promotional purposes, creating new collective works, for resale or redistribution to servers or lists, or reuse of any copyrighted component of this work in other works." 


\title{
Data Visualisation of Complex Adaptive Systems
}

\author{
Satyendra Sinha and Zenon Chaczko \\ University of Technology Sydney \\ Sydney, Australia \\ satyendra.sinha@student.uts.edu.au and zenon.chaczko@uts.edu.au
}

\begin{abstract}
In Complex Adaptive Systems, the complexity of a construct is predicated by its heterogenic entities and the dynamic nature of their interactions. These interactions are often non-probablistic and triggered as a consequence of potentially multiple previous effects, which can lead to emergent behaviours of the overall system not present in the individual sub-systems. These systems are ubiquitous, not only in natural habitats and within living organisms but also in human-made constructs such as communities, organisations and technology that often have a life of their own. Human-made constructs are particularly interesting due to the data generated that can be unstructured, multidimensional and dynamic, often containing outlier data that may be considered anomalous but can still have critical impact.

Complex adaptive data or data generated from Complex Adaptive Systems have interesting ramifications, particularly for data visualisation. Due to the unpredictable nature of complex adaptive data well known forms of data visualisation such as bar charts, scatter plots and heatmaps are inadequate in effectively communicating the data stories in Complex Adaptive Systems. There is a need for more advanced data visualisations that caters for complex adaptive data as it can serve as an educational and decision-making tool for experimentation of ideas in systems that are complex and adaptive.
\end{abstract}

Keywords-complex; adaptive; data; visualisation;

\section{INTRODUCTION}

The study of Complex Adaptive Systems (CAS) involves appreciating the serendipitous nature of interactions between heterogeneous entities within a system. Especially, in terms of microscopic or local behaviours leading to vastly different behaviours at the macroscopic or global level.

The interactions between heterogenous entities within a CAS is rich, dynamic and unpredictable, akin to a conversation amongst a group of friends. The data reflecting these interactions is of particular interest as data points are almost whimsical as the schema can be dynamic or static, structured or unstructured. Being able to monitor the flow of data in a CAS posits an interesting data visualisation exercise.

There have been many recent efforts into capitalising on cloud and ubiquitous infrastructures for more advanced data visualisation [1]. However in this case, being able to visualise the effects of local interactions and trace the effects on a global scope is a very niche and unique challenge, as well as being the objective of this study. In order to overcome such a challenge a model is required that can be drawn from to visualise emergent behaviours and patterns in CAS. The proposal in this study is to draw inspiration from the endocrine system; an instance of CAS that is present in almost all multi-cellular organisms.

The endocrine system is made up of organs that secrete hormones, which moderate the internal environment in response to the external environment. The mechanistic conceptions of the endocrine system has been used extensively in several projects and are collectively known as Artificial Endocrine Systems (AES), a particular branch of biomimetics, as they are artificial uses of the principles of the endocrine system. AES can be thought of as a specific kind of CAS, since biology has already demonstrated the success of such systems, and as such serves as inspiration for data visualisation of complex adaptive data.

The next section of this paper provides a review of scenarios where modelling using CAS was useful in reducing the complexity of their respective scenarios. The following section dicusses particular properties of CAS, which are also present in AES, that prove challenging to visualise. Finally, the discussion and conclusion section refer to the lessons learned in this paper.

\section{Review of Modelling By Complex AdAPtive SYSTEMS}

Complex Adaptive Systems (CAS) are characterised by self-organisation, emergent behaviour and complex interaction patterns. While initially a framework to represent the complex faceted interactions in nature and biology, These traits make CAS a versatile methodology for modeeling inorganic systems in various domains. Some of which are exemplified below.

City planning is a difficult and intricate venture with various complexities such as population densities, climate change, socio-economic conditions as just a few elements that make it a complex system [2]. Nel et al. suggests viewing cities as a social-ecological system (SES) that have scale in the form of individual buildings to city regions, while also having properties of emergent behaviour as the demands of population growth and need for infrastructure maintenance increases over time. By virtue of SES the transition to viewing cities as CAS requires identifying the properties and asking the appropriate questions to determine the boundaries as demonstrated in the table figure 1.

Another example of CAS can be expressed in the push and pull of sustainable logging of forests against local markets, which are influenced by globalisation of trade [3]. The traditional approach was based around viewing forests as inherently stable has been proven to be unsustainable 
Table 1. Proposed questions to assist in identifying the properties of CAS.

\begin{tabular}{|c|c|}
\hline CAS Property & Question \\
\hline Agents and networks & $\begin{array}{l}\text { Who or what has agency (i.e. people, households, firms)? Who or what makes the system work? Who } \\
\text { are the stakeholders? Are there any aggregate agents (i.e. firms, households)? What activities do } \\
\text { the agents perform? Where are the agents? Do the agents move? If so how? How are the agents } \\
\text { connected? What type of connections exists? What moves between agents (i.e. information, } \\
\text { money, goods)? How are the networks between agents structured? }\end{array}$ \\
\hline Adaptation & What has changed? How has it changed? What caused the change? \\
\hline $\begin{array}{l}\text { Self-organisation and } \\
\text { emergence }\end{array}$ & $\begin{array}{l}\text { What are the trends or patterns observed at a system level that is a result of collective actions taken } \\
\text { by individuals (i.e. population growth, traffic)? }\end{array}$ \\
\hline Hierarchies & $\begin{array}{l}\text { How does the system being studied fit into the bigger picture? Can the system being studied be } \\
\text { broken into clearly defined sub-components/elements (i.e. social, economic, institutional } \\
\text { elements) What events from the levels above and below have impacted on the studied system? }\end{array}$ \\
\hline Diversity & $\begin{array}{l}\text { Is there any diversity in activity (i.e. land use)? Are there differences in the agents that make up the } \\
\text { system (i.e. differences in race, culture, religion)? What, if any, are the differences in types of } \\
\text { agents? Are there any differences in the composition of the agents or aggregate agents? Is there a } \\
\text { diversity of routes through which the flows move (i.e. diverse transportation systems)? }\end{array}$ \\
\hline Redundancy & $\begin{array}{l}\text { Will the system continue to function if a part of it collapse (i.e. power blackouts or accidents on key } \\
\text { though routes)? Are there back-ups for different parts or functions of the system? Can a function } \\
\text { of the system be performed by another part or agent(s)? }\end{array}$ \\
\hline \multicolumn{2}{|c|}{ How do these properties show themselves spatially? } \\
\hline
\end{tabular}

Figure 1. Table from [2] for asking questions to identify CAS properties

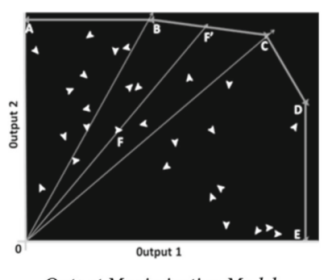

Output Maximization Model

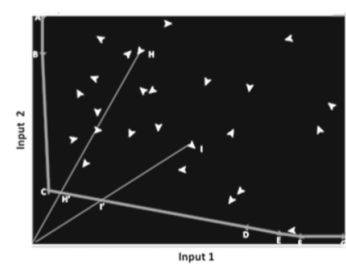

Input Minimization Model

Figure 2. Agent based Complex Adaptive Systems modelling in [4]

and in many cases irresponsible as forest ecosystems were degrading over time. By taking a CAS approach the uncertainty and dynamic nature of forests can be appreciated. Furthermore intricacies such as biodiversity, epigenetics and socio-environmental systems are considered as part of a CAS model.

CAS can also be used to model abstract yet measurable concepts such as productive efficiency analysis [4]. Decision-making-units (DMUs) are agents that interact within a system based on certain given rules as per the flocking metaphor to achieve mutual protection and reduce risks. These behaviours are represented in agent-based modelling simulations such as the output maximization and input minimization models as shown in 2 .

Blended learning is another area that CAS modelling has been applied as a systems approach [5]. By applying a framework of Complex Adaptive Blended Learning Systems (CABLS), as shown in figure 3, the various heterogeneous elements of learning such as teachers, technology, content, institution, learner/student and learning support can be appreciated. By identifying the relationships and the emergent behaviour of students learning, a deeper understanding of blended learning can be appreciated.

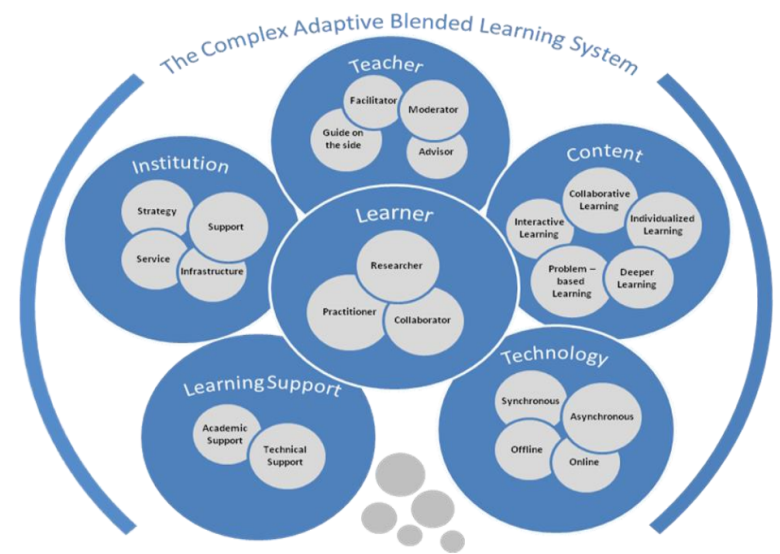

Figure 3. CABLS as highlighted in [5]

\section{Visualising Properties of COMPLEX ADAPTIVE DATA}

In the previous position paper [6], we identified three properties in Artificial Endocrine Systems (AES) that can be considered to be pertinent to human-made Complex Adaptive Systems (CAS) such as Internet of Things infrastructure, which are self-organisation, synchronisation and cascading effects. In the endocrine system each of these properties serve a unique function in maintaining balance. The endocrine system has been used to draw inspiration in multiple biomimicry based research. In this study, we focus on properties mentioned earlier as a means to visualise the data generated within CAS i.e complex adaptive data.

\section{A. Self-organisation}

Self-organisation shown below in figure 4 is the grouping of related nodes in a system based on a particular 
function. Each node can contain specialised yet related functionality to other nodes in the system. In complex adaptive data, this refers to the multi-dimensionality of data points.

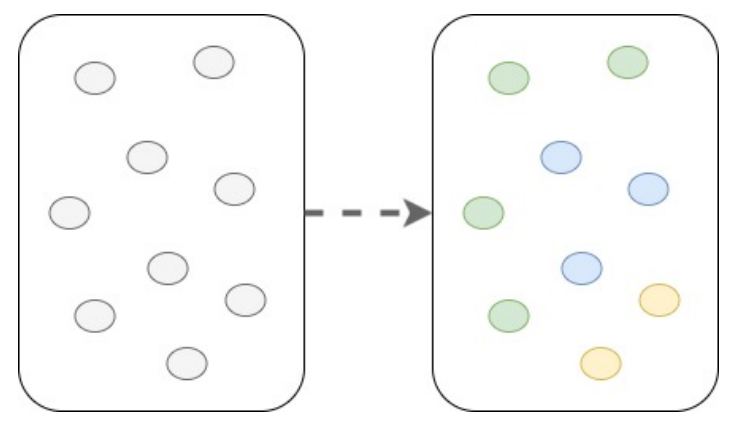

Figure 4. Self-organisation

There are many examples of self-organising strategies in literature that are used in technology from robotics to hardware to networking. An interesting example can be found in [7] that describes a system for self-organising of internet protocol television grid where quality of the signal is affected by the number, distribution and type of end user devices.

Data analytics and visualisation of internet protocol television data would be a challenging task as the number of devices are expontentially increasing and the protocol needs to maintain functionality against this load. Observing and comparing usage density and viewing quality in different regions would be interesting data points.

\section{B. Synchronisation}

Synchronisation as found in figure 5 refers to the feedback loops of data passing between nodes. Here nodes pass information between each other and influence the local behaviour of the system. Local changes can have cascading effects throughout the system that can have global ramifications, as discussed in the next subsection. In complex adaptive systems, synchronisation refers to the rich interactions that occur between data points.

Receiving and storing sensor data to maintain a virtual model of the internal and external environment of vehicles requires synchronisation of sensor data points against the model as demonstrated in [8]. These data points can serve

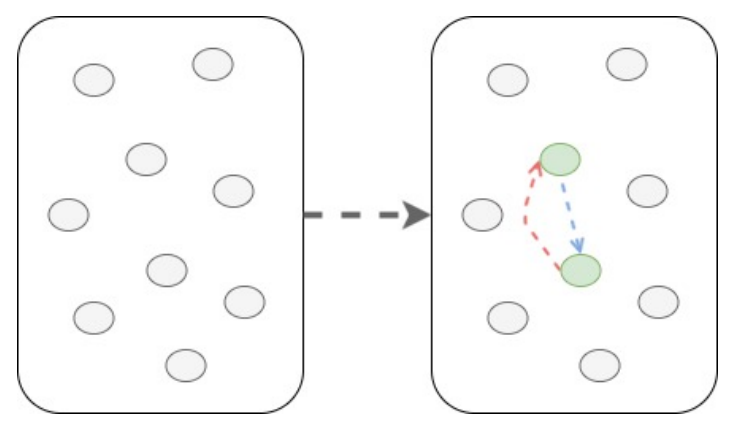

Figure 5. Synchronisation

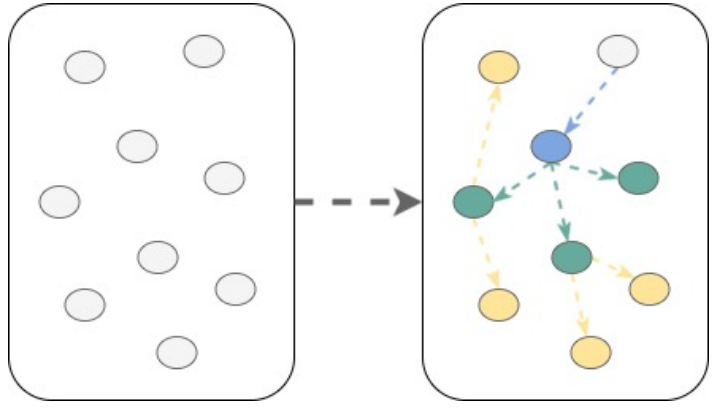

Figure 6. Cascading effects

as $\operatorname{logs}$ to parse through when required for insurance claims for accidents to verify eye witness reports.

While data synchronisation of converting analog signals to digital signals to map a model is useful in this scenario, additional utility could be provided by detecting or visualising the synchronisation that occurs between data points within this virtual model. Sensory information from the external environment is bound to affect the internal environment, being able to detect and map this could provide much more information than generating traditional visualisations out of logs.

\section{Cascading Effects}

Cascading effects refers to the primary and secondary effects that are triggered as a response to an initial effect. Figure 6 shows how one data point can trigger effects further along the system. Here seemingly minor local interactions can have significant impacts globally across the network leading to the emergence behaviours and patterns complex adaptive systems are known for.

[9] describes a solution to calculating cascading failure relationships between correlated anomalies by organising network performance metrics into historical anomaly dependency data. As the transmission of data across data centers grows exponentially so too does the complexity of the system, which makes anomalies and catastrophic failures that much more difficult to discern as there maybe multiple and often hidden sources of failure. Collating anomaly dependency data to detect cascading interactions is a useful excercise in monitoring and predicting potential sources of failure. In this case, building intuitive visualisations out of dependency data can lead to proactive investigations of potential error prone regions of data centers.

\section{DISCUSSION AND CONCLUSION}

Each of the properties above demonstrates that the endocrine system can inspire further work in data visualisation of complex adaptive systems. In biomimetics, the study of mimicking nature as a means of solving complex human problems, there is a specialised branch that draws inspiration from the endocrine system known as artificial endocrine systems. The lessons learned from observing artificial endocrine systems as a precursor to visualising complex adaptive data be summarised in table I above. 


\begin{tabular}{|l|l|l|l|}
\hline Property & Endocrine Feature & Summary & Technology Scenarios \\
\hline Self-organisation & Endocrine Organs & Grouping of related nodes in a system & Internet protocol television grid [7] \\
\hline Synchronisation & Homeostasis & Data feedback loops & vehicle sensor information [8] \\
\hline Cascading Effects & Co-occurrence of endocrine interactions & propagating effects of response & Historical anomaly dependency data [9] \\
\hline
\end{tabular}

Table I

SUMMARY OF LESSONS LEARNED FOR SELF-ORGANISATION, SYNCHRONISATION AND CASCADING EFFECTS

There are many domains that would benefit from being able to visualise complex adaptive data. For instance, neuroscience is a field where research and data is being generated at an alarming rate and being able to categorise it into information is proving challenging as [10] indicates. Data visualisation is identified as one of the key ingredients in being able to track progress in the neuroscience field.

Beyond the scientific community, many multi-national businesses such as Amazon, Google, IBM and Microsoft are deeply investing into research and development for data visualisation and there has been much advancement over the years. The value that data visualisation brings is that provides a visual sandbox for experimenting with the effects of data, and this is already being done by multinational businesses. However little has been done in the way of complex adaptive data visualisation, and that is the niche that this study aims to address.

The value this study will bring to research and education is that we want to be able to experiment with the data, models and systems that we have at our disposal in a safe and inexpensive manner. The future is going to be rife with systems that are complex and adaptive. The convergence of big data, artificial intelligence, internet of things and other related technologies will make the future essentially unpredictable. At the very least we should be able to 'see' what the immediate effects are, what the cascading effects will be, experiment with these data points and make intuitive decisions in between.

\section{REFERENCES}

[1] L. Ferreira, G. D. Putnik, N. Lopes, W. Garcia, M. M. Cruz-Cunha, H. Castro, M. L. Varela, J. M. Moura, V. Shah, C. Alves et al., "Disruptive data visualization towards zerodefects diagnostics," Procedia CIRP, vol. 67, pp. 374-379, 2018.

[2] D. Nel, C. du Plessis, and K. Landman, "Planning for dynamic cities: introducing a framework to understand urban change from a complex adaptive systems approach," International Planning Studies, pp. 1-14, 2018.

[3] C. Messier, K. Puettmann, R. Chazdon, K. Andersson, V. Angers, L. Brotons, E. Filotas, R. Tittler, L. Parrott, and S. Levin, "From management to stewardship: viewing forests as complex adaptive systems in an uncertain world," Conservation Letters, vol. 8, no. 5, pp. 368-377, 2015.

[4] F. L. Dougherty, N. P. Ambler, and K. P. Triantis, "A complex adaptive systems approach for productive efficiency analysis: building blocks and associative inferences," Annals of Operations Research, vol. 250, no. 1, pp. 45-63, 2017.
[5] Y. Wang, X. Han, and J. Yang, "Revisiting the blended learning literature: Using a complex adaptive systems framework." Journal of Educational Technology \& Society, vol. 18, no. 2, 2015.

[6] S. Sinha and Z. Chaczko, "Concepts and observations in artificial endocrine systems for iot infrastructure," in Systems Engineering (ICSEng), 2017 25th International Conference on. IEEE, 2017, pp. 427-430.

[7] H. A. Salama, J. Dunne, P. B. French, J. P. Galvin Jr, and P. J. O'sullivan, "System for self organization of an internet protocol television grid," Jan. 3 2017, uS Patent 9,537,707.

[8] T. Binion, J. Harr, B. Fields, S. Cielocha, and S. J. Balbach, "Synchronization of vehicle sensor information," Jun. 7 2016, uS Patent 9,361,650.

[9] A. Sasturkar and N. Alan, "Organizing network performance metrics into historical anomaly dependency data," Apr. 25 2017, uS Patent 9,632,858.

[10] R. L. Goldstone, F. Pestilli, and K. Börner, "Self-portraits of the brain: cognitive science, data visualization, and communicating brain structure and function," Trends in cognitive sciences, vol. 19, no. 8, pp. 462-474, 2015. 